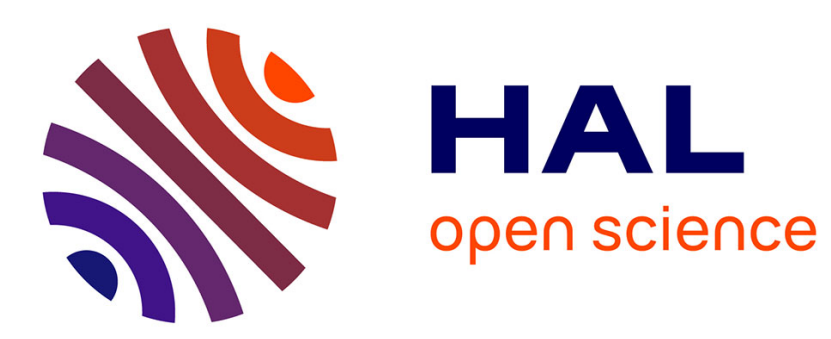

\title{
Almost Global Synchronization in Radial Multi-Machine Power Systems
}

Johannes Schiffer, Denis Efimov, Romeo Ortega

\section{To cite this version:}

Johannes Schiffer, Denis Efimov, Romeo Ortega. Almost Global Synchronization in Radial MultiMachine Power Systems. 57th IEEE Conference on Decision and Control (CDC 2018), Dec 2018, Miami Beach, FL, United States. hal-01888553

\section{HAL Id: hal-01888553 \\ https://hal.inria.fr/hal-01888553}

Submitted on 5 Oct 2018

HAL is a multi-disciplinary open access archive for the deposit and dissemination of scientific research documents, whether they are published or not. The documents may come from teaching and research institutions in France or abroad, or from public or private research centers.
L'archive ouverte pluridisciplinaire HAL, est destinée au dépôt et à la diffusion de documents scientifiques de niveau recherche, publiés ou non, émanant des établissements d'enseignement et de recherche français ou étrangers, des laboratoires publics ou privés. 


\title{
Almost Global Synchronization in Radial Multi-Machine Power Systems
}

\author{
Johannes Schiffer, Denis Efimov and Romeo Ortega
}

\begin{abstract}
Sufficient conditions for almost global synchronization of second-order multi-machine power systems with radial topology are provided. The analysis is based on the recently proposed multivariable cell structure approach using Leonov functions-an extension of the powerful cell structure principle developed by Leonov and Noldus to nonlinear systems, the dynamics of which are periodic with respect to several state variables and possess multiple invariant solutions. The efficiency of the derived conditions is illustrated via a numerical example.
\end{abstract}

\section{INTRODUCTION}

\section{A. Motivation}

Undoubtly, power systems are one of the most complex man-made engineering networks [1]. At the same time, our modern societies crucially rely on a secure and reliable electricity supply, i.e., on a well-functioning power system. Yet, in addition to their mere extension in terms of size and number of components, power systems exhibit a rich nonlinear behavior and are permanently subjected to disturbances [1]-[3]. Therefore, the task of instantaneously supplying the customer's load demand, while ensuring a safe and stable system operation is very challenging. This challenge has constantly been increasing in the past years as more and more (deterministic) conventional generation units are replaced by (volatile) renewable energy sources [4]. As a consequence, the number of transient disturbances acting on the power network has increased drastically and the system is operated under higher stress [4].

One of the most important problems in power system operation is to-following a disturbance-maintain all rotational generation units in synchrony, i.e., spinning at the same speed [2]. If large disturbances (e.g., a short circuit) are considered, this is called the transient stability problem [2]. As a consequence of a large disturbance, the resulting system's trajectories are highly influenced by the nonlinear system behavior and the generator rotor angles may exhibit severe changes [2]. This directly implies that a local analysis is, in general, insufficient to fully reveal the systems' stability properties and dynamic behavior. Motivated by this, the present paper is devoted to the global transient stability

J. Schiffer is with Brandenburgische Technische Universität, Germany, schiffereb-tu.de

D. Efimov is with Inria, Univ. Lille, CNRS, UMR 9189 - CRIStAL, France and Department of Control Systems and Informatics, University ITMO, Russia, Denis.Efimoveinria.fr

R. Ortega is with Laboratoire des Signaux et Systémes, École Supérieure d'Electricité (SUPELEC), France, ortega@lss.supelec.fr

This work was partially supported by the Government of Russian Federation (Grant 08-08) and the Ministry of Education and Science of Russian Federation (Project 14.Z50.31.0031).

J. Schiffer acknowledges funding from the European Union's Horizon 2020 research and innovation programme under the Marie SkłodowskaCurie grant agreement No. 734832 analysis of a power system, in which synchronous generators (SGs) are represented by the standard swing equation.

\section{B. Existing Literature}

The available approaches in the literature usually depart from the following setup [2], [5]. Consider a power system and denote its state vector by $x$. Suppose the system is operating in a synchronized state $x_{\text {pre }}$. Then a fault occurs and is cleared after an interval of time. The post-fault power system might be different from the pre-fault system, since the faulted component may have been disconnected. Assume the post-fault system possesses a synchronized state $x_{\text {post }}$. The key questions addressed in transient stability studies are if $x_{\text {post }}$ is attractive and if the initial condition $x_{0}$ of the postfault system is within the attractor of $x_{\text {post }}$.

There are two main approaches for transient stability studies: time domain simulations and analytic Lyapunovbased approaches (also called direct energy methods) [6]. Compared to a simulation-based stability assessment, Lyapunov methods have the advantages that system stability can be verified in a rigorous manner without explicitly solving the system dynamics and without screening over a large range of, possibly harmless, contingencies [6]. To this end, there exists a variety of techniques, such as the identification of the (closest) unstable equilibrium point (UEP) [7] or algorithms based on convex optimization [8] as well as consensus-inspired approaches [9].

All the results discussed above pursue a local analysis of the system based on the following conjecture. Although a power system usually admits many synchronized states [10], the standard implicit assumption adopted in the literature is that the state $x_{\text {post }}$ of interest is the one "closest" to the prefault one $x_{\text {pre }}$. Thus, also $x_{0}$ will presumably be "close" to 1 $x_{\text {post }}$, hence justifying the focus on local analyses [5].

Despite this (well-known) observation, there are only few works addressing a global analysis of the power system dynamics. Some global properties of power systems with generators modeled by the swing equation have been reported in [5]. By using more detailed SG models, a global stability analysis is performed in [11]. Yet, the result critically relies on the construction of a very specific synchronized state and a particular value for the mechanical torque of each SG in the system, both of which limit the practicality of the approach. Related results have been obtained under less stringent assumptions in [12]-[16], but these are limited to the single-machine-infinite-bus scenario.

\section{Contributions}

The main contribution of the present paper is to provide sufficient conditions for almost global synchronization of

\footnotetext{
${ }^{1}$ Note that, in general, $x_{0} \neq x_{\text {pre }}$.
} 
a multi-machine power system with radial topology and in which SGs are represented by the standard swing equation. This is done by using the recently developed framework of multivariable cell structures and the concept of a Leonov function [17], [18]. The work in [17], [18] is inspired by the cell structure framework introduced for systems with a scalar periodic variable by Leonov and co-workers [19], [20] as well as in [21]. Compared to the original cell structure framework, the approach in [17], [18] is applicable to nonlinear systems, the dynamics of which are periodic with respect to several state variables and which possess multiple invariant solutions. Clearly, both latter properties are inherent features of multi-machine power systems.

Compared to standard Lyapunov theory, the use of Leonov functions permits to relax the usual sign definiteness requirements on the Lyapunov function and its time-derivative by exploiting the periodicity of the system. This relaxation is essential to provide conditions for global boundedness of trajectories in the present paper. By using this result together with LaSalle's invariance principle [22], we establish almost global asymptotic stability of the attractive equilibrium set of the power system, i.e., we show that for all initial conditions, except a set of measure zero, the solutions of the power system asymptotically converge to a stable equilibrium.

At this point, it seems convenient to discuss an important technical issue with regard to our main result. As any stability analysis (numerical or analytical), the present analysis is model-based. We are aware that the employed power system model is simplified and does not necessarily capture the global behavior of a true physical power system. Nonetheless, we believe the present analysis makes a significant contribution towards a more complete understanding of the synchronization problem in large-scale power systems by providing a very different (global) perspective on the problem compared to the available literature. In addition, the results are directly applicable to the synchronization problem of second-order Kuramoto oscillators [23], [24] and our employed approach has also the potential to be used-with appropriate modifications - in the global analysis of more generic complex oscillator networks [25].

Notation. We define the sets $\mathbb{R}_{\geq 0}:=\{x \in \mathbb{R} \mid x \geq 0\}$, $\mathbb{R}_{>0}:=\{x \in \mathbb{R} \mid x>0\}$ and $\bar{S}:=[0,2 \pi)$. The set of nonnegative integers is denoted by $\mathbb{Z}_{\geq 0}$. For a set $\mathcal{V},|\mathcal{V}|$ denotes its cardinality and $[\mathcal{V}]^{k}$ denotes the set of all subsets of $\mathcal{V}$ that contain $k$ elements. For a set of, possibly unordered, positive natural numbers $\mathcal{V}=\{l, k, \ldots, n\}$, the short-hand $i \sim \mathcal{V}$ denotes $i=l, k, \ldots, n$. Given a positive integer $n$, we use $\underline{0}_{n}$ to denote the vector of all zeros, $\mathbb{1}_{n}$ the vector with all ones and $I_{n}$ the $n \times n$ identity matrix. Let $x=\operatorname{col}\left(x_{1}, \ldots, x_{n}\right) \in \mathbb{R}^{n}$ denote a column vector with entries $x_{i} \in \mathbb{R}$. Let $\operatorname{diag}\left(a_{i}\right) \in \mathbb{R}^{n \times n}$ denote a diagonal matrix with entries $a_{i} \in \mathbb{R}$. For a matrix $A \in \mathbb{R}^{n \times n}, \lambda_{\min }(A)$ denotes its minimum eigenvalue. For a function $f: \mathbb{R}^{n} \rightarrow \mathbb{R}$, $\nabla f$ denotes its gradient and $\nabla^{2} f$ its Hessian. We employ the notation $\nabla f\left(x^{*}\right):=\left.\nabla f(x)\right|_{x=x^{*}}$ and $\nabla^{2} f\left(x^{*}\right):=$ $\left.\nabla^{2} f(x)\right|_{x=x^{*}}$. Let $j$ denote the imaginary unit. For $x \in \mathbb{R}^{n}$, the element-wise sine, cosine, and tanh functions are denoted by $\sin (x) \in[-1,1]^{n}, \cos (x) \in[-1,1]^{n}$ and $\tanh (x) \in$ $[-1,1]^{n}$, respectively. Also, $\tanh ^{2}(x)$ denotes the square function applied element-wise to $\tanh (x)$. Furthermore, $|x|=\sqrt{x^{\top} x}$ denotes the usual Euclidean norm of a vector $x \in \mathbb{R}^{n}$ and $|x|_{\infty}=\max _{i}\left|x_{i}\right|$ its infinity norm.

\section{Preliminaries}

\section{A. Power System Model}

We consider a radial multi-machine power system model with $N>1$ nodes. The topology of the electrical network is described by an undirected and connected graph $\mathcal{G}=(\mathcal{N}, \mathcal{E})$, where the set of nodes is denoted by $\mathcal{N}=\{1,2, \ldots, N\}$ and the set of edges (representing power lines) by $\mathcal{E}=$ $\left\{e_{1}, \ldots, e_{N-1}\right\}$, see [26]. Furthermore, by associating an arbitrary ordering to the edges, we introduce the node-edge incidence matrix $\mathcal{B} \in \mathbb{R}^{N \times N-1}$, the entries of which are defined as $b_{i l}=1$ if node $i$ is the source of the $l$-th edge $e_{l}$, $b_{i l}=-1$ if $i$ is the sink of $e_{l}$ and $b_{i l}=0$ otherwise.

To each node $i \in \mathcal{N}$, we associate a phase angle $\theta_{i}$ : $\mathbb{R}_{\geq 0} \rightarrow \mathbb{R}$ and the corresponding electrical frequency $\omega_{i}=$ $\dot{\theta}_{i}$. In order to establish a fundamental claim in this paper, namely convergence of bounded solutions, it is necessary to define the angles $\theta_{i}$ on the real line, rather than (as usual) on the torus ${ }^{2}$. Yet, this implies that the angles are not bounded a priori, making it necessary to establish boundedness of trajectories separately, which is done in Section III-C.

Following the standard approach in transient stability studies [1], [3], [28], we assume that the voltage amplitudes at all nodes are positive real constants, i.e., $V_{i} \in \mathbb{R}_{>0}$ and that the line admittances are purely inductive. Hence, if node $i \in \mathcal{N}$ is connected to node $k \in \mathcal{N}$, this is represented by the nonzero susceptance $B_{i k}=B_{k i} \in \mathbb{R}_{<0}$. If these nodes are not connected via a power line, then $B_{i k}=0$. The set of neighbors of a node $i \in \mathcal{N}$ is denoted by $\mathcal{N}_{i}:=\left\{k \mid k \in \mathcal{N}, k \neq i, B_{i k} \neq 0\right\}$. With these considerations, the active power flow $P_{i}: \mathbb{R}^{\left|\mathcal{N}_{i}\right|} \rightarrow \mathbb{R}$ at node $i$ is given $\mathrm{by}^{3}$ [3]

$$
P_{i}=G_{i i} V_{i}^{2}+\sum_{k \sim \mathcal{N}_{i}} V_{i} V_{k}\left|B_{i k}\right| \sin \left(\theta_{i k}\right),
$$

where we have introduced the short-hand $\theta_{i k}=\theta_{i}-\theta_{k}$ and $G_{i i} \in \mathbb{R}_{\geq 0}$ represents the shunt conductance at node $i$.

Furthermore, the dynamics of the unit at the $i$-th node, $i \in \mathcal{N}$, are given by the standard swing equation [1], [3]

$$
\begin{aligned}
\dot{\theta}_{i} & =\omega_{i}, \\
M_{i} \dot{\omega}_{i} & =-D_{i} \omega_{i}-P_{i}+P_{i}^{d},
\end{aligned}
$$

where $M_{i} \in \mathbb{R}_{>0}$ is the inertia constant, $D_{i} \in \mathbb{R}_{>0}$ is the damping and droop coefficient, $P_{i}^{d} \in \mathbb{R}$ is the active power setpoint and the active power flow $P_{i}$ is given by (II.1).

In order to write the system (II.1), (II.2), $i \sim \mathcal{N}$, compactly, we introduce the matrices

$$
M:=\operatorname{diag}\left(M_{i}\right) \in \mathbb{R}_{>0}^{N \times N}, D:=\operatorname{diag}\left(D_{i}\right) \in \mathbb{R}_{>0}^{N \times N}
$$

${ }^{2}$ Defining the phase angles in Euclidean space is also a common step in local Lyapunov-based stability analysis of power systems [8], [27].

${ }^{3}$ To simplify notation the time argument of all signals is omitted in the sequel. 
and the vectors

$$
\begin{aligned}
& \theta:=\operatorname{col}\left(\theta_{i}\right) \in \mathbb{R}^{N}, \omega:=\operatorname{col}\left(\omega_{i}\right) \in \mathbb{R}^{N}, \\
& P^{\text {net }}:=\operatorname{col}\left(P_{i}^{d}-G_{i i} V_{i}^{2}\right) \in \mathbb{R}^{N} .
\end{aligned}
$$

With $a_{m}=V_{i} V_{k}\left|B_{i k}\right|, m=1, \ldots, N-1$, we define the diagonal matrix of power line weights

$$
A:=\operatorname{diag}\left(a_{m}\right) \in \mathbb{R}_{>0}^{N-1}
$$

and the potential function $U: \mathbb{R}^{N} \rightarrow \mathbb{R}$,

$$
\begin{aligned}
U(\theta) & :=-\sum_{\{i, k\} \in \mathcal{N} \times \mathcal{N}} V_{i} V_{k}\left|B_{i k}\right| \cos \left(\theta_{i k}\right) \\
& =-\mathbb{1}_{N-1}^{\top} A \cos \left(\mathcal{B}^{\top} \theta\right) .
\end{aligned}
$$

The potential $U(\theta)$ and its gradient,

$$
\nabla U(\theta)=\mathcal{B} A \sin \left(\mathcal{B}^{\top} \theta\right),
$$

possess certain symmetry properties, i.e., for any $\alpha \in \mathbb{R}$,

$$
U\left(\theta+\alpha \mathbb{1}_{N}\right)=U(\theta), \quad \nabla U\left(\theta+\alpha \mathbb{1}_{N}\right)=\nabla U(\theta) .
$$

These symmetry properties arise from the fact that the power flows (II.1) only depend upon angle differences. Furthermore, since $\mathbb{1}_{N}^{\top} \mathcal{B}=\underline{0}_{N}$,

$$
\mathbb{1}_{N}^{\top} \nabla U(\theta)=0 .
$$

Then, the system (II.1), (II.2), $i \sim \mathcal{N}$, can be written as

$$
\begin{aligned}
\dot{\theta} & =\omega, \\
M \dot{\omega} & =-D \omega-\nabla U(\theta)+P^{\text {net }} .
\end{aligned}
$$

Clearly, the dynamics (II. 8 ) are $2 \pi$-periodic in the variables $\theta_{i}, i=1, \ldots, N$, and hence satisfy [18, Assumption 1].

Remark 2.1: In addition to a bulk power system, the model (II.8) can also represent a microgrid with constant voltage amplitudes and power setpoints $P^{d}$, see [26], [29], as well as a second-order Kuramoto model [23]. For $N=1$ the model (II.8) reduces to a nonlinear pendulum.

\section{B. Synchronized Motions and Equilibria}

We employ the definition below to characterize desired invariant solutions of the system (II.8), see also [29].

Definition 2.2: The system (II.8) admits a synchronized motion if it has an invariant solution of the form

$$
\theta^{s}(t)=\omega^{s} t+\theta_{0}^{s}, \quad \omega^{s}=\omega^{*} \mathbb{1}_{N}, \quad \forall t \geq 0,
$$

where $\omega^{*} \in \mathbb{R}$ and $\theta_{0}^{s} \in \mathbb{R}^{n}$ such that

$$
\left|\theta_{0, i}^{s}-\theta_{0, k}^{s}\right|<\frac{\pi}{2} \quad \forall i \in \mathcal{N}, \forall k \in \mathcal{N}_{i} .
$$

Note that the properties (II.6) have the following implication for synchronized motions of the system (II.8). If the system (II.8) possesses a synchronized motion $\theta^{s}(t)=\omega^{s} t+$ $\theta_{0}^{s}+\alpha \mathbb{1}_{N}, \omega^{s}=\omega^{*} \mathbb{1}_{N}$ with $\alpha=0$, then it always admits an infinite number of synchronized solutions for $\alpha \in \mathbb{R}$. From (II.7) and (II.8), it follows that for $\dot{\omega}^{s}=\underline{0}_{N}$ [29],

$$
\omega^{*}=\frac{\mathbb{1}_{N}^{\top} P^{\mathrm{net}}}{\mathbb{1}_{N}^{\top} D \mathbb{1}_{N}} .
$$

Therefore, $\omega^{*}$ is uniquely defined by $P^{\text {net }}$ and $D$.
Motivated by these observations and inspired by [30]-[32], we introduce the new variable

$$
\eta=\mathcal{B}^{\top} \theta \in \mathbb{R}^{N-1},
$$

where we recall that $\mathcal{B}$ is the network incidence matrix and $\mathcal{E}$ the set of power lines. Thus, $\eta$ is a projection of $\theta$ on the subspace orthogonal to $\mathbb{1}_{N}$ and defines the phase angle differences between the nodes. Furthermore, with the change of variables (II.10) it also follows from (II.4) that

$$
\begin{aligned}
U(\eta) & =-\mathbb{1}_{N-1}^{\top} A \cos (\eta), \\
\nabla U(\eta) & =A \sin (\eta), \quad \nabla^{2} U(\eta)=A \cos (\eta),
\end{aligned}
$$

and thus from (II.5) that

$$
\nabla U(\theta)=\mathcal{B} A \sin \left(\mathcal{B}^{\top} \theta\right)=\mathcal{B} \nabla U(\eta) .
$$

In the new coordinates, the dynamics (II.8) are given by

$$
\begin{aligned}
\dot{\eta} & =\mathcal{B}^{\top} \omega, \\
M \dot{\omega} & =-D \omega-\mathcal{B} \nabla U(\eta)+P^{\text {net }} .
\end{aligned}
$$

Clearly, appearance of a synchronized motion of the system (II.8) corresponds to an equilibrium of (II.12), i.e.,

$\eta^{*}=\mathcal{B}^{\top}\left(\theta_{0}^{s}+\omega^{s} t+\alpha \mathbb{1}_{N}\right)=\mathcal{B}^{\top}\left(\theta_{0}^{s}+\left(\omega^{*} t+\alpha\right) \mathbb{1}_{N}\right)=\mathcal{B}^{\top} \theta_{0}^{s}$ and asymptotic stability of $\operatorname{col}\left(\eta^{*}, \omega^{s}\right)$ implies asymptotic convergence of the solutions $\operatorname{col}(\theta, \omega)$ to $\operatorname{col}\left(\theta^{s}, \omega^{s}\right)$ up to a constant uniform shift in all angles $\theta_{a v} \mathbb{1}_{N}$.

\section{Almost Global Synchronization of Multi-Machine Power Systems - A LeOnov FUNCTION APPROACH}

This section is dedicated to the analysis of global synchronization in multi-machine power systems. Our analysis is conducted by employing the recently proposed framework of Leonov functions [17], [18].

\section{A. Error Coordinates}

Recall from (II.9) that the synchronization frequency $\omega^{*}$ is uniquely defined. However, the question whether the system (II.12) possesses one or more equilibrium solutions (modulo $2 \pi$ ) is very hard to answer in a general setting. In fact, that problem is identical to the problem of existence of solutions to the nonlinear active power flow problem [10], [33] and to that of determining the equilibria of a network of Kuramoto oscillators [34] — both of which are long-standing active research areas on their own, see [34]. Furthermore, it follows from [31, Theorem 2] that any equilibrium satisfying $\left|\eta_{0 i}^{*}\right|<\frac{\pi}{2}, i=1, \ldots, N-1$, is locally asymptotically stable.

As a consequence of the abovementioned facts and as existence of isolated equilibria is a natural prerequisite for any stability analysis, we make the following assumption.

Assumption 3.1: The system (II.12) only possesses isolated equilibria $\operatorname{col}\left(\eta^{*}, \omega^{*} \mathbb{1}_{N}\right) \in \mathbb{R}^{2 N-1}$, at least one of which is locally asymptotically stable. The Jacobian matrix of the dynamics (III.3) evaluated at any unstable equilibrium point has at least one eigenvalue with positive real part.

With Assumption 3.1, we denote an asymptotically stable equilibrium point of the system (II.12) by $\operatorname{col}\left(\eta^{*}, \mathbb{1}_{N} \omega^{*}\right)$ and introduce the error states

$$
\tilde{\eta}(t):=\eta(t)-\eta^{*}, \tilde{\omega}(t):=\omega(t)-\mathbb{1}_{N} \omega^{*} .
$$


Furthermore, by introducing the short-hand

$$
\zeta(\tilde{\eta}):=\nabla U\left(\tilde{\eta}+\eta^{*}\right)-\nabla U\left(\eta^{*}\right),
$$

the system (II.12) becomes in error coordinates

$$
\begin{aligned}
\dot{\tilde{\eta}} & =\mathcal{B}^{\top} \tilde{\omega}, \\
M \dot{\tilde{\omega}} & =-D \tilde{\omega}-\mathcal{B} \zeta(\tilde{\eta}),
\end{aligned}
$$

the nominal equilibrium of which is now shifted to the origin and, because of Assumption 3.1, is isolated and asymptotically stable. The remainder of this section is devoted to the stability analysis of equilibria of this system.

\section{B. Leonov Function Candidate}

The notion of a Leonov function is introduced following [17], [18]. For its presentation, we define two auxiliary sets:

$$
\begin{aligned}
\mathcal{W} & :=\left\{\operatorname{col}(\tilde{\eta}, \tilde{\omega}) \in \mathbb{R}^{2 N-1}:|\tilde{\eta}|_{\infty}=c\right\}, \pi \leq c<2 \pi, \\
\mathcal{U} & :=\cup_{r \in \mathbf{Z}_{\geq 0}} \mathcal{U}_{r}, \\
\mathcal{U}_{r} & :=\left\{\operatorname{col}(\tilde{\eta}, \tilde{\omega}) \in \mathbb{R}^{2 N-1}: \tilde{\omega}=\underline{0}_{N},|\tilde{\eta}|_{\infty}=2 r \pi\right\} .
\end{aligned}
$$

Then, a Leonov function for the system (III.3) is defined as follows [17], [18].

Definition 3.2 ([18]): A $\mathcal{C}^{1}$ function $V: \mathbb{R}^{2 N-1} \rightarrow \mathbb{R}$ is a Leonov function for the system (III.3) if there exist a constant $g \geq 0$, functions $\alpha \in \mathcal{K}_{\infty}, \psi \in \mathcal{K}$ and a continuous function $\lambda: \mathbb{R} \rightarrow \mathbb{R}$, satisfying $\lambda(0)=0$ and $\lambda(s) s>0$ for all $s \neq 0$, such that

$$
\begin{aligned}
& \alpha(|\tilde{\omega}|)-\psi(|\tilde{\eta}|)-g \leq V(\tilde{\eta}, \tilde{\omega}) \forall \operatorname{col}(\tilde{\eta}, \tilde{\omega}) \in \mathbb{R}^{2 N-1}, \\
& \inf _{\operatorname{col}(\tilde{\eta}, \tilde{\omega}) \in \mathcal{W}} V(\tilde{\eta}, \tilde{\omega})>0, \sup _{\operatorname{col}(\tilde{\eta}, \tilde{\omega}) \in \mathcal{U}} V(\tilde{\eta}, \tilde{\omega}) \leq 0
\end{aligned}
$$

and the following dissipation inequality holds:

$$
\dot{V}+\lambda(V) \leq 0 \quad \forall \operatorname{col}(\tilde{\eta}, \tilde{\omega}) \in \mathbb{R}^{2 N-1} .
$$

From (III.5) and (III.6), we see that the sign definiteness requirements of a Leonov function are relaxed compared to the standard Lyapunov function [22], because the function $V$ in Definition 3.2 does not have to be positive definite with respect to the variable $\tilde{\eta}$, i.e., the variable with respect to which the dynamics (III.3) are periodic. Furthermore, the time-derivative of $V$ only needs to be negative definite for positive values of $V$. See [17], [18] for further details.

In addition, the requirements on (III.6) have been further relaxed in [18, Corollary 3]. This result is used to establish the main result of the present paper and hence recalled here. For this purpose, we introduce the following sets:

$$
\begin{aligned}
\Omega & =\left\{\operatorname{col}(\tilde{\eta}, \tilde{\omega}) \in \mathbb{R}^{2 N-1}: V \leq 0\right\}, \\
\Omega_{\varepsilon, c}^{\prime} & =\left\{\operatorname{col}(\tilde{\eta}, \tilde{\omega}) \in \mathbb{R}^{2 N-1}: V \leq \varepsilon,|\tilde{\eta}|_{\infty}<c\right\}, \\
\mathcal{Z} & =\left\{\operatorname{col}(\tilde{\eta}, \tilde{\omega}) \in \mathbb{R}^{2 N-1}:|\tilde{\omega}|>\xi\right\},
\end{aligned}
$$

with $c$ defined in (III.4) and for some $\varepsilon \in \mathbb{R}_{>0}$ and $\xi \in \mathbb{R}_{>0}$.

Corollary 3.3 ([18]): Suppose that there exists a Leonov function $V: \mathbb{R}^{2 N-1} \rightarrow \mathbb{R}$ for the system (III.3), such that $\sup _{\tilde{\eta} \in \mathbb{R}^{N-1}} \psi(|\tilde{\eta}|)<+\infty$ and the inequality (III.6) is verified only for $\operatorname{col}(\tilde{\eta}, \tilde{\omega}) \in\left(\mathbb{R}^{2 N-1} \backslash \Omega\right) \cap\left(\mathcal{Z} \cup \Omega_{\varepsilon, c}^{\prime}\right)$. Then for all initial conditions $\operatorname{col}(\tilde{\eta}(0), \tilde{\omega}(0)) \in \mathbb{R}^{2 N-1}$ the corresponding trajectories $\operatorname{col}(\tilde{\eta}, \tilde{\omega})$ are bounded $\forall t \geq 0$.
The Leonov function candidate employed in our subsequent global analysis of invariant solutions of the dynamics (III.3) is introduced below. To this end, let

$$
h(\tilde{\eta}, \tilde{\omega}):=\alpha D \tilde{\omega}+\mathcal{B} \zeta(\tilde{\eta}),
$$

with $\alpha \in[0,1], \zeta(\tilde{\eta})$ defined in (III.2) and

$\frac{d}{d t} h=-\alpha D M^{-1} h+\left(\alpha(\alpha-1) D^{2} M^{-1}+\mathcal{B} S\left(\tilde{\eta}+\eta^{*}\right)\right) \tilde{\omega}$,

$S\left(\tilde{\eta}+\eta^{*}\right):=\nabla^{2} U\left(\tilde{\eta}+\eta^{*}\right) \mathcal{B}^{\top}=A \cos \left(\tilde{\eta}+\eta^{*}\right) \mathcal{B}^{\top}$.

Let $\kappa \in \mathbb{R}_{\geq 0}$ be a parameter and $\Phi \in \mathbb{R}^{N \times N}, \Phi=\Phi^{\top}>0$, be a design matrix. Then our proposed Leonov function candidate for the system (III.3) is given by

$$
\begin{aligned}
& V(\tilde{\eta}, \tilde{\omega}):=\tilde{\omega}^{\top} M \tilde{\omega}+h^{\top}(\tilde{\eta}, \tilde{\omega}) \Phi h(\tilde{\eta}, \tilde{\omega})-\kappa \\
& \\
&+2\left[U\left(\tilde{\eta}+\eta^{*}\right)-U\left(\eta^{*}\right)-\nabla U^{\top}\left(\eta^{*}\right) \tanh (\tilde{\eta})\right] \\
&= {\left[\begin{array}{c}
\mathcal{B} \zeta(\tilde{\eta}) \\
\tilde{\omega}
\end{array}\right]^{\top} \underbrace{\left[\begin{array}{cc}
\Phi & \alpha \Phi D \\
\alpha D \Phi & M+\alpha^{2} D \Phi D
\end{array}\right]}_{:=\Psi}\left[\begin{array}{c}
\mathcal{B} \zeta(\tilde{\eta}) \\
\tilde{\omega}
\end{array}\right]-\kappa } \\
&+2\left[U\left(\tilde{\eta}+\eta^{*}\right)-U\left(\eta^{*}\right)-\nabla U^{\top}\left(\eta^{*}\right) \tanh (\tilde{\eta})\right] .
\end{aligned}
$$

We also make use of this lower bound for $V$ in the sequel:

$$
\begin{aligned}
\underline{V}(\tilde{\eta})= & 2\left[U\left(\tilde{\eta}+\eta^{*}\right)-U\left(\eta^{*}\right)-\nabla U^{\top}\left(\eta^{*}\right) \tanh (\tilde{\eta})\right] \\
& +\lambda_{\min }(\Psi)|\mathcal{B} \zeta|^{2}-\kappa \leq V, \quad \forall(\tilde{\eta}, \tilde{\omega}) \in \mathbb{R}^{2 N-1},
\end{aligned}
$$

and, in particular, of its behavior in the set

$$
\underline{\Omega}=\left\{\operatorname{col}(\tilde{\eta}, \tilde{\omega}) \in \mathbb{R}^{2 N-1}: \underline{V} \leq 0,0<|\tilde{\eta}|_{\infty} \leq c\right\} .
$$

\section{A Condition for Global Boundedness of Solutions}

A sufficient condition for global boundedness of trajectories is presented by deriving conditions under which $V$ in (III.10) is a Leonov function for the dynamics (III.3). To streamline the presentation of our result the following assumption is needed.

Assumption 3.4: Recall the sets $\mathcal{W}$ and $\underline{\Omega}$ and the function $V$ defined in (III.4), (III.12) and (III.11). Consider the matrix $\bar{Q}(\tilde{\eta})$ given in (III.13). There exist parameters $c \in[\pi, 2 \pi)$, $\Phi>0, \alpha \in[0,1], \nu>0, \beta>0$ and $\mu>0$, such that

$$
\begin{gathered}
Q(\tilde{\eta})>0, \quad \inf _{\operatorname{col}(\tilde{\eta}, \tilde{\omega}) \in \mathcal{W}} \underline{V}(\tilde{\eta})>0, \\
\sup _{\left(\tilde{\eta}, \underline{,}_{N}\right) \in \underline{\Omega}}-\beta\left|\mathcal{B} \zeta\left(\tilde{\eta}+\eta^{*}\right)\right|^{2}+\frac{1}{\nu}\left|\mathcal{B} \operatorname{diag}\left(\tanh ^{2}(\tilde{\eta})\right) \nabla U\left(\eta^{*}\right)\right|^{2}<0 .
\end{gathered}
$$

Proposition 3.5: Consider the system (III.3) with Assumptions 3.1 and 3.4. Select

$$
\kappa=2 \sum_{i=1}^{N-1}\left|a_{i} \sin \left(\eta_{i}^{*}\right)\right| .
$$

Then, the function $V$ in (III.10) is a Leonov function for the system (III.3). Furthermore, all solutions of the system (III.3) are bounded. 


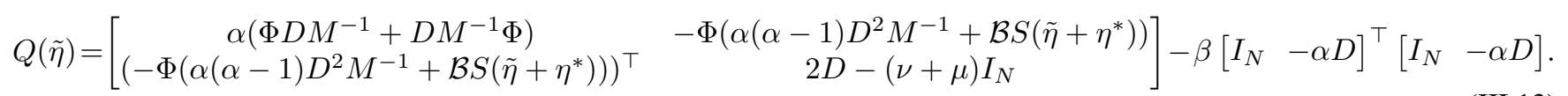

Proof: The claim is established by invoking Corollary 3.3. Recall the sets $\mathcal{W}$ and $\mathcal{U}$ defined in (III.4). Since $\tanh (\tilde{\eta}) \in[-1,1]^{N-1}$, by choosing $\kappa$ as specified in (III.15), we ensure that $\sup _{\operatorname{col}(\tilde{\eta}, \tilde{\omega}) \in \mathcal{U}} V \leq 0$. By assump-

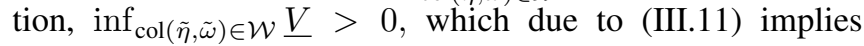

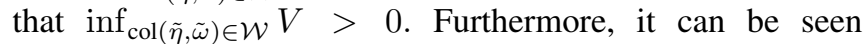
from (III.10) in a straightforward manner that the required functions $\alpha \in \mathcal{K}_{\infty}$ and $\psi \in \mathcal{K}$ as well as the constant $g \geq 0$ exist for the proposed $V$. Hence, the conditions in (III.5) are satisfied. Moreover, since $U\left(\tilde{\eta}+\eta^{*}\right), \tanh (\tilde{\eta})$ and $\zeta\left(\tilde{\eta}+\eta^{*}\right)$ are bounded functions, the additional requirement $\sup _{\tilde{\eta} \in \mathbb{R}^{N-1}} \psi(|\tilde{\eta}|)<+\infty$ of Corollary 3.3 is satisfied.

Next, with (III.9) and

$$
\frac{d}{d t} \tanh (\tilde{\eta})=\operatorname{diag}\left(\mathbb{1}_{N-1}-\tanh ^{2}(\tilde{\eta})\right) \dot{\tilde{\eta}}
$$

we have that

$$
\begin{aligned}
\dot{V} \leq & -\left[\begin{array}{c}
h \\
\tilde{\omega}
\end{array}\right]^{\top} Q(\tilde{\eta})\left[\begin{array}{c}
h \\
\tilde{\omega}
\end{array}\right]-\mu\left|\tilde{\omega}^{2}\right|-\beta|\mathcal{B} \zeta|^{2} \\
& +\frac{1}{\nu}\left|\mathcal{B} \operatorname{diag}\left(\tanh ^{2}(\tilde{\eta})\right) \nabla U\left(\eta^{*}\right)\right|^{2} \\
\leq & -\mu\left|\tilde{\omega}^{2}\right|-\beta|\mathcal{B} \zeta|^{2}+\frac{1}{\nu}\left|\mathcal{B} \operatorname{diag}\left(\tanh ^{2}(\tilde{\eta})\right) \nabla U\left(\eta^{*}\right)\right|^{2},
\end{aligned}
$$

where $Q(\tilde{\eta})$ is given in (III.13), $\nu, \mu$ as well as $\beta$ are positive parameters and the last inequality follows since $Q(\tilde{\eta})>0$ by assumption.

Recall that both $\zeta$ and tanh are bounded functions, $\mu>0$ and $\left|\mathcal{B} \nabla U\left(\eta^{*}\right)\right|$ is a constant. Hence, it is evident that there exist $\xi>0$ and $\chi>0$, such that $\dot{V}+\chi V \leq 0$ for all $\operatorname{col}(\tilde{\eta}, \tilde{\omega}) \in \mathcal{Z}$ defined in (III.7). Assumption 3.4 and the facts that $\dot{V}\left(\tilde{\eta}, \underline{0}_{N}\right) \geq \dot{V}(\tilde{\eta}, \tilde{\omega})$ for all $\operatorname{col}(\tilde{\eta}, \tilde{\omega}) \in \mathbb{R}^{2 N-1}$ and $\dot{V} \leq 0$ for all $\operatorname{col}\left(\underline{0}_{N-1}, \tilde{\omega}\right) \in \mathbb{R}^{2 N-1}$ imply that there exist (sufficiently small) parameters $\varepsilon>0$ and $\chi>0$, such that $\dot{V} \leq-\chi V$ for all

$$
(\tilde{\eta}, \tilde{\omega}) \in\left\{\operatorname{col}(\tilde{\eta}, \tilde{\omega}) \in \mathbb{R}^{2 N-1}: \underline{V} \leq V \leq \varepsilon,|\tilde{\eta}|_{\infty}<c\right\},
$$

with $\underline{V}$ defined in (III.11). This, in turn, implies the existence of $\Omega_{\varepsilon, c}^{\prime}$ with the desired properties.

Thus, all conditions of Corollary 3.3 are satisfied. Hence, $V$ is a Leonov function for the system (III.3) and all solutions $\operatorname{col}(\tilde{\eta}, \tilde{\omega}) \in \mathbb{R}^{2 N-1}$ are bounded for all $t \geq 0$.

Remark 3.6: Physically, the term $\mathcal{B} \nabla U\left(\eta^{*}\right)$ in Assumption 3.4 corresponds to the stationary network power flows. Thus, the conditions for global boundedness of trajectories in Proposition 3.5 are more likely to be satisfied in lightly loaded operating conditions. This seems reasonable from a practical point of view.

\section{Main Synchronization Result}

Recall that with Assumption 3.1, all equilibria of the system (III.3) are isolated and at least one of these equilibria is asymptotically stable. As the system (III.3) is continuous, this implies necessarily that some of the remaining equilibria are unstable. For an illustration see, e.g., the related numerical experiments in [34]. Denote by $\mathcal{X}$ the set of asymptotically stable equilibria of the system (III.3).

The result below shows that, in addition to boundedness of solutions, the conditions of Proposition 3.5 also imply almost global asymptotic stability of the set $\mathcal{X}$.

Theorem 3.7: Consider the system (III.3) with Assumptions 3.1 and 3.4. The set $\mathcal{X}$ is almost globally asymptotically stable, i.e., for all initial conditions, except a set of measure zero, the solutions of the system (III.3) asymptotically converge to a point in $\mathcal{X}$.

Proof: The proof is established by using LaSalle's invariance principle [22] together with the function

$$
W(\tilde{\eta}, \tilde{\omega})=\frac{1}{2} \tilde{\omega}^{\top} M \tilde{\omega}+U\left(\tilde{\eta}+\eta^{*}\right)-\nabla U^{\top}\left(\eta^{*}\right) \tilde{\eta}
$$

and following the steps of [15, Theorem 1]. The details are omitted for space reasons.

\section{NumericAl EXAMPLE}

The analysis is illustrated via an example derived from the IEEE 9 bus test system with three generators [28, Chapters 2 and 9]. We consider the network corresponding to the pre-fault configuration as described in [28, Chapter 9]. In accordance with our assumptions, we neglect the transfer conductances in the off-diagonal entries of the reduced admittance matrix. Furthermore, the network topology in [28, Chapter 9] is meshed. To obtain a radial topology, we eliminate the (smallest) off-diagonal entry of the admittance matrix, i.e., the one between generators 2 and 3. Following standard practice, we set the droop gains to $D_{i}=\frac{1}{0.05}$ [pu] (with respect to the rated machine powers). All other system parameters are as given in [28, Chapter 9].

The conditions in Assumption 3.4 are evaluated for a range of operating points. At first we determine the parameters $\alpha$, $\beta, \nu$ and $\Phi$, such that $Q(\tilde{\eta}) \leq 0$. From Assumption 3.4 it is evident that we seek to maximize $\beta$ and $\nu$. This is done via a polytopic approach and by implementing the corresponding matrix inequalities in Yalmip [35] with $\mu=0$. With the given system data, we obtain $\beta=2.6 \cdot 10^{-2}$ and $\nu=33.76$

Next we evaluate the feasibility of the remaining two conditions in Assumption 3.4 for a wide range of different operating points. We find that the conditions are feasible for values up to $\left|\eta_{1}^{*}\right|=28^{\circ}$ and $\left|\eta_{2}^{*}\right|=25^{\circ}$. This shows that a reasonable range of operating points can be guaranteed to be almost globally asymptotically stable with the conditions of Proposition 3.5. In Fig. 1 the contour plots corresponding to the functions appearing in the second and third conditions of Assumption 3.4 are shown for an exemplary operating point with $\eta^{*}=\operatorname{col}\left(22^{\circ},-25^{\circ}\right)$. It can be seen that for $\mathcal{W}$ in (III.4) with $c=\pi$, Assumption 3.4 is satisfied.

\section{CONCLUSIONS}

In contrast to the prevailing local synchronization analysis in the literature, we have derived sufficient conditions for 


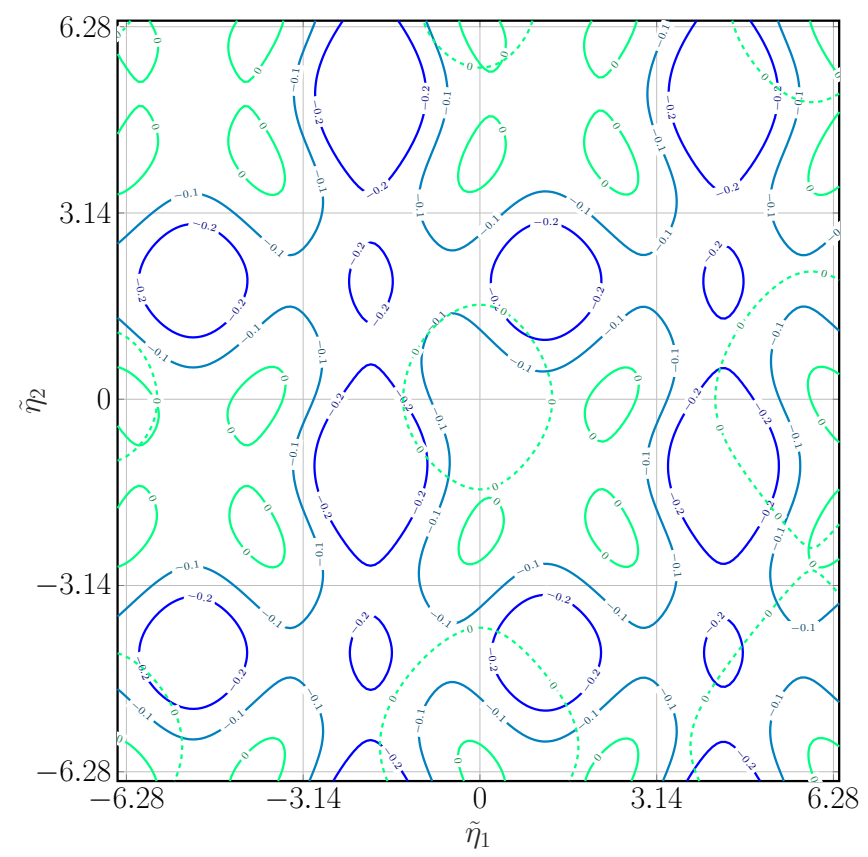

Fig. 1. Contour plot of $-\beta\left|\mathcal{B} \zeta\left(\tilde{\eta}+\eta^{*}\right)\right|^{2}+\frac{1}{\nu}\left|\mathcal{B} \operatorname{diag}\left(\tanh ^{2}(\tilde{\eta})\right) \nabla U\left(\eta^{*}\right)\right|^{2}$ for $|\tilde{\eta}|_{\infty} \leq 2 \pi$ and $\eta^{*}=\operatorname{col}\left(22^{\circ},-25^{\circ}\right)$. The dashed curves represent the level set $V=0$. The plots show that with $c=\pi$ the conditions on $\Omega$ and $\mathcal{W}$ in Assumption 3.4 are satisfied.

almost global synchronization in multi-machine power systems with radial topology. The result has been established by combining LaSalle's invariance principle with the recently developed concept of Leonov functions [17], [18]. The analysis has been illustrated via numerical experiments and it has been shown that the proposed conditions can be verified in a reasonably broad range of operating scenarios.

Usually, LaSalle-based convergence claims do not inherently provide some kind of robustness guarantees. In addition, the model employed in the analysis is a simplified representation of a true power system. Hence, two natural extensions of the presented results are to provide additional robustness measures to account for model uncertainties and extend the analysis to more detailed models with meshed topologies. Both directions are currently under investigation.

\section{REFERENCES}

[1] J. Machowski, J. Bialek, and J. Bumby, Power system dynamics: stability and control. J.Wiley \& Sons, 2008.

[2] P. Kundur et al., "Definition and classification of power system stability IEEE/CIGRE joint task force on stability terms and definitions," IEEE Trans. on Power Systems, vol. 19, no. 3, pp. 1387-1401, 2004.

[3] P. Kundur, Power system stability and control. McGraw-Hill, 1994.

[4] W. Winter, K. Elkington, G. Bareux, and J. Kostevc, "Pushing the limits: Europe's new grid: Innovative tools to combat transmission bottlenecks and reduced inertia," IEEE Power and Energy Magazine, vol. 13 , no. 1 , pp. $60-74,2015$.

[5] A. Arapostathis, S. Sastry, and P. Varaiya, "Global analysis of swing dynamics," IEEE Trans. on Circuits and Systems, vol. 29, no. 10, pp. 673-679, 1982.

[6] M. Pavella, D. Ernst, and D. Ruiz-Vega, Transient stability of power systems: a unified approach to assessment and control. Springer Science \& Business Media, 2012.

[7] H.-D. Chang, C.-C. Chu, and G. Cauley, "Direct stability analysis of electric power systems using energy functions: theory, applications, and perspective," Proceedings of the IEEE, vol. 83, no. 11, pp. 1497$1529,1995$.

[8] T. L. Vu and K. Turitsyn, "Lyapunov functions family approach to transient stability assessment," IEEE Trans. on Power Systems, vol. 31, no. 2 , pp. 1269-1277, 2016.
[9] F. Dörfler and F. Bullo, "Synchronization and transient stability in power networks and non-uniform Kuramoto oscillators," SIAM Journal on Control and Optimization, vol. 50, no. 3, pp. 1616-1642, 2012.

[10] J. Baillieul and C. Byrnes, "Geometric critical point analysis of lossless power system models," IEEE Trans. on Circuits and Systems, vol. 29 , no. 11 , pp. 724-737, 1982.

[11] S. Caliskan and P. Tabuada, "Compositional transient stability analysis of multimachine power networks," IEEE Trans. on Control of Network Systems, vol. 1, no. 1, pp. 4-14, March 2014.

[12] V. Natarajan and G. Weiss, "Almost global asymptotic stability of a constant field current synchronous machine connected to an infinite bus," in $C D C, 2014$, pp. 3272-3279.

[13] _ - "A method for proving the global stability of a synchronous generator connected to an infinite bus," in 28th Convention of Electrical \& Electronics Engineers in Israel, 2014, pp. 1-5.

[14] N. Barabanov, J. Schiffer, R. Ortega, and D. Efimov, "Almost global attractivity of a synchronous generator connected to an infinite bus," in $C D C, 2016$, pp. 4130-4135.

[15] — - "Conditions for almost global attractivity of a synchronous generator connected to an infinite bus," IEEE Trans. on Aut. Control, vol. 62, no. 10, pp. 4905-4916, Oct 2017

[16] J. Schiffer, D. Efimov, R. Ortega, and N. Barabanov, "An input-to-state stability approach to verify almost global stability of a synchronousmachine- infinite-bus system," Philosophical Trans. of the Royal Society of London. A, pp. 1887-1895, Aug. 2017.

[17] D. Efimov and J. Schiffer, "A new criterion for boundedness of solutions for a class of periodic systems," in ECC, 2018, pp. 16421647.

[18] — "On boundedness of solutions of periodic systems: a multivariable cell structure approach," Available online at HAL-Inria (http://hal.inria.fr/), 2018.

[19] G. Leonov, "On the boundedness of the trajectories of phase systems," Siberian Mathematical Journal, vol. 15, no. 3, pp. 491-495, 1974

[20] —_, "Phase synchronisation. theory and applications," Nonlinear Systems: Frequency and Matrix Inequalities, 2008.

[21] E. J. Noldus, "New direct Lyapunov-type method for studying synchronization problems," Automatica, vol. 13, no. 2, pp. 139-151, 1977.

[22] A. van der Schaft, L2-gain and passivity techniques in nonlinear control. Springer, 2000

[23] F. Dörfler and F. Bullo, "Synchronization in complex networks of phase oscillators: A survey," Automatica, vol. 50, no. 6, pp. 15391564,2014

[24] F. A. Rodrigues, T. K. D. Peron, P. Ji, and J. Kurths, "The Kuramoto model in complex networks," Physics Rep., vol. 610, pp. 1-98, 2016.

[25] Y. Tang, F. Qian, H. Gao, and J. Kurths, "Synchronization in complex networks and its application-a survey of recent advances and challenges," Annual Reviews in Ctrl., vol. 38, no. 2, pp. 184-198, 2014.

[26] J. Schiffer, D. Zonetti, R. Ortega, A. M. Stanković, T. Sezi, and J. Raisch, "A survey on modeling of microgrids - from fundamental physics to phasors and voltage sources," Automatica, vol. 74, pp. 135$150,2016$.

[27] H.-D. Chiang, F. Wu, and P. Varaiya, "A BCU method for direct analysis of power system transient stability," IEEE Trans. on Power Systems, vol. 9, no. 3, pp. $1194-1208$, aug 1994.

[28] P. Anderson and A. Fouad, Power System Control and Stability. J.Wiley \& Sons, 2002

[29] J. Schiffer, R. Ortega, A. Astolfi, J. Raisch, and T. Sezi, "Conditions for stability of droop-controlled inverter-based microgrids," Automatica, vol. 50, no. 10 , pp. $2457-2469,2014$

[30] M. Arcak, "Passivity as a design tool for group coordination," IEEE Trans. on Aut. Control, vol. 52, no. 8, pp. 1380-1390, 2007.

[31] T. Stegink, C. De Persis, and A. van der Schaft, "A unifying energybased approach to stability of power grids with market dynamics," IEEE Trans. on Aut. Control, vol. 62, no. 6, pp. 2612-2622, 2017.

[32] S. Trip, M. Bürger, and C. De Persis, "An internal model approach to (optimal) frequency regulation in power grids with time-varying voltages," Automatica, vol. 64, pp. 240-253, 2016.

[33] A. Araposthatis, S. Sastry, and P. Varaiya, "Analysis of power-flow equation," International Journal of Electrical Power and Energy Systems, vol. 3, no. 3, pp. 115 - 126, 1981.

[34] D. Mehta, N. S. Daleo, F. Dörfler, and J. D. Hauenstein, "Algebraic geometrization of the kuramoto model: Equilibria and stability analysis," Chaos: An Interdisciplinary Journal of Nonlinear Science, vol. 25 no. 5 , p. 053103,2015

[35] J. Löfberg, "YALMIP : a toolbox for modeling and optimization in MATLAB," in IEEE International Symposium on Computer Aided Control Systems Design, sept. 2004, pp. 284 -289. 